\title{
The deep red state of photosystem II in Cyanidioschyzon merolae
}

\author{
J. LANGLEY*, J. MORTON*, R. PURCHASE*, L. TIAN**, L. SHEN**, G. HAN*, J.-R. SHEN*,***, \\ and E. KRAUSZ ${ }^{*+}$ \\ Research School of Chemistry, Australian National University, Canberra, Australia* \\ Photosynthesis Research Center, Key Laboratory of Photobiology, Institute of Botany, the Chinese Academy of Sciences, \\ Beijing 100093, China ${ }^{* *}$ \\ Research Institute for Interdisciplinary Science and Graduate School of Natural Science and Technology, Okayama \\ University, Okayama 700-8530, Japan ${ }^{* * *}$
}

\begin{abstract}
We identified and characterised the deep red state (DRS), an optically-absorbing charge transfer state of PSII, which lies at lower energy than P680, in the red algae Cyanidioschyzon merolae by means of low temperature absorption and magnetic circular dichroism spectroscopies. The photoactive DRS has been previously studied in PSII of the higher plant Spinacia oleracea, and in the cyanobacterium Thermosynechococcus vulcanus. We found the DRS in PSII of C. merolae has similar spectral properties. Treatment of PSII with dithionite leads to reduction of cytochrome (cyt) $b_{559}$ and the $P s b V$ based cyt $c_{550}$ as well as the disassembly of the oxygen-evolving complex. Whereas the overall visible absorption spectrum of PSII was little affected, the DRS absorption in the reduced sample was no longer seen. This bleaching of the DRS is discussed in terms of a corresponding lack of a DRS feature in D1D2/cyt $b_{559}$ reaction centre preparations of PSII.
\end{abstract}

Additional key words: optical spectra; photosynthesis.

The splitting of water in nature is catalysed by the PSII enzyme. Light absorption is followed by transfer of excitation energy to the reaction centre (RC) of PSII, which induces charge separation and the creation of the powerful oxidant P680.+. The oxidation of two molecules of water to molecular oxygen occurs through a step-wise oxidation of the oxygen-evolving complex (OEC) via a redox active tyrosine residue $\left(\mathrm{Tyr}_{\mathrm{Z}}\right)$. After four oxidation events, the OEC extracts four electrons from two molecules of water, releasing molecular oxygen whilst returning the $\mathrm{OEC}$ to its least oxidised configuration. The catalytic cycle of PSII is described in terms of a series of intermediate $\left(\mathrm{S}_{\mathrm{i}}\right)$ states, where the subscript $\mathrm{i}$ denotes the number of stored oxidising equivalents. The process is considered to be very similar in all oxygenic photosynthetic organisms.

Great strides have been made in a broad range of studies on PSII, with the emphasis often being on the OEC.
The clear goal is the elucidation of the detailed mechanism of water oxidation (Shen 2015), which will enable the development of artificial water-splitting catalysts. Many recent studies have concentrated on the prokaryotic thermophile $T$. vulcanus and its close relative T. elongatus. The structure of $T$. vulcanus has been determined to atomic (1.9 Å) resolution (Umena et al. 2011, Suga et al. 2015).

The only eukaryotic PSII for which a crystal structure has been obtained (Adachi et al. 2009, Ago et al. 2016) is the red algae Cyanidium caldarium. This structure has lower $(2.76 \AA)$ resolution than that of $T$. vulcanus, yet is clear in showing the organisation of PSII to be largely conserved between the two organisms. Red algae appear to be unique in containing a protein subunit PsbQ' which has low homology to the PsbQ subunit found in higher plants (Enami et al. 2008, Ifuku 2015). The water exchange kinetics of the $\mathrm{OEC}$ in $C$. merolae, which is

Received 14 June 2017, accepted 21 August 2017, published as online-first 3 January 2018.

+Corresponding author; fax: +61-2-6125-0750, e-mail: elmars.krausz@anu.edu.au

Abbreviations: Chl - chlorophyll; cyt - cytochrome; DRS - deep red state; MCD - magnetic circular dichroism; OD - optical density; $\mathrm{OEC}$ - oxygen-evolving complex; $\mathrm{RC}$ - reaction centre.

Acknowledgements: E. Krausz would like to thank Govindjee for bringing the work eventually published in reference (Pettai et al. 2005) to his attention whilst both attended the Photosynthesis Congress in Montreal in 2004. We recognise the support of the Australian Research Council through grants DP110104565 and DP 150103137 (E. Krausz), a Strategic Priority Research Program of CAS (XDB17030100) and a CAS Key Research Project for Frontier Science (QYZDY-SSW-SMC003) of China, and MEXT/JSPS of Japan through a Grant-in-Aid for Specially Promoted Research No. 24000018 (J.-R.S.). 
closely related to Cyanidium caldarium, have been shown to be significantly different from those of other studied organisms (Nilsson et al. 2014) and point to significant (but perhaps minor) differences in the mechanism of water oxidation in OEC in red algae.

The RC present in PSII core complexes derived from $S$. oleracea and $T$. vulcanus exhibits a broad, relatively weak absorption feature that extends to $730 \mathrm{~nm}$ at $2 \mathrm{~K}$ (Hughes et al. 2006, Morton et al. 2014, Morton et al. 2015). Low temperature illumination of this DRS of PSII, at wavelengths longer than $700 \mathrm{~nm}$, leads to charge separation and secondary donor formation in a majority of PSII centres. The absorption band is attributed to an optically accessible charge transfer state of the special pair $\mathrm{P}_{\mathrm{D} 1}-\mathrm{P}_{\mathrm{D} 2}$, and provides an alternative charge separation mechanism to the $\mathrm{Chl}_{\mathrm{D} 1}$ based $\mathrm{P} 680$ process. The DRS also exhibits broad emission, peaking at $780 \mathrm{~nm}$, in both S. oleracea and T. vulcanus (Morton et al. 2014). The OEC of PSII possesses weak absorption in the 700-900 nm spectral range and thus overlaps with absorption of the DRS. The OEC of T. vulcanus has been found to exhibit well structured and distinct magnetic circular dichroism (MCD) in the $\mathrm{S}_{1}, \mathrm{~S}_{2}$, and $\mathrm{S}_{3}$ states attributable to $\mathrm{Mn}(\mathrm{IV})$ spin flip ${ }^{4} \mathrm{~A} \rightarrow{ }^{2} \mathrm{E}$ absorption in $\mathrm{Mn}_{4} \mathrm{CaO}_{5}$ catalytic cluster of the OEC (Morton et al. 2015, Morton et al. 2017).

Parallel to the initial discovery of the low temperature spectral characteristics of the DRS were experiments made on plant leaves of Helianthus annuus and Phaseolus vulgaris at room temperature, which showed that oxygenic photosynthesis could occur using light with wavelengths as long as $780 \mathrm{~nm}$ (Pettai et al. 2005). The existence of a similar low-energy, optically accessible charge transfer state has also been demonstrated in PSI (Schlodder 2014, Morton 2015). The varied phenomenologies of low energy states in photosynthesis is of significant current interest (Reimers et al. 2016).

PSII from $C$. merolae was prepared using methods previously described (Adachi et al. 2009, Ago et al. 2016) and measured on a laboratory-constructed spectrometer system, which has the important advantage of being able to measure simultaneous absorption and MCD spectra at cryogenic temperatures with high sensitivity and precision (Krausz 2013). Samples were prepared to possess either a high or a low optical density (OD) by mixing PSII cores of different concentration with a $45 \%(\mathrm{v} / \mathrm{v})$ glycol/glycerol $(1: 1, \mathrm{v} / \mathrm{v})$ glassing medium. High OD samples were prepared using $\sim 3.3 \mathrm{mg}(\mathrm{Chl} a) \mathrm{ml}^{-1}$ concentration and were measured in a 1-mm path length hydrophobic cell. Low OD samples were prepared using either $\sim 3.3$ or $\sim 0.18 \mathrm{mg}(\mathrm{Chl} a) \mathrm{ml}^{-1}$ and measured in a $0.07-\mathrm{mm}$ path length or a 1-mm path length hydrophobic cell, respectively. These were loaded and handled in low light before being dark-adapted for $5 \mathrm{~min}$ and subsequently cooled to $4 \mathrm{~K}$ over a $\sim 1 \mathrm{~min}$ period. Absorption and MCD spectra were recorded at $1.8 \mathrm{~K}$ and $40 \mathrm{~K}$. Measurements were also made on dithionite-reduced samples for which $5 \mu \mathrm{l}$ of a $\sim 22 \mathrm{mg}$ (dithionite) $\mathrm{ml}^{-1}$ was added to $54 \mu \mathrm{l}$ of cryoprotectant solution immediately before the addition of PSII cores.

Low-temperature photo-illumination experiments were also performed. For these experiments, very low measurement light fluences were used, ensuring that the absorption spectra recorded before and after illumination of the sample with intense green light were not affected by the measurement light itself. Full details of the protocols used are described by Hughes et al. (2006). These nonactinic measurements enable precise identification of the Pheo $_{\mathrm{D} 1} \mathrm{Q}_{\mathrm{x}}$ absorption, which undergoes a strong electrochromic shift (historically called the 'C550' shift) (Butler and Okayama 1971). Upon photo-illumination a range of other shifts and bleaches associated with the reduction of the plastoquinone $\mathrm{Q}_{\mathrm{A}}$ to its anion $\left(\mathrm{Q}_{\mathrm{A}}^{-}\right)$and the creation of a secondary donor occur (Hughes et al. 2010). This secondary donor may be a Chl $a$, a reduced cyt $b_{559}$ or a carotenoid in the RC.

The absorption changes seen upon photo-illumination shown in Fig. 1 (central thin grey line) are very well resolved and parallel corresponding measurements made on PSII in S. oleracea and T. elongatus (Krausz et al. 2005, Hughes et al. 2010). The observed highly structured features enable immediate identification of the Pheo ${ }_{D 1} Q_{x}$ transition at $547.5 \mathrm{~nm}$ and the $\mathrm{Chl}_{\mathrm{D} 1} \mathrm{Q}_{\mathrm{y}}$ exciton at $684.2 \mathrm{~nm}$ by means of their distinctive electrochromic shifts (Hughes et al. 2008). These shifts are induced by illumination of the low temperature sample, which causes charge separation and subsequent metastable $\mathrm{Q}_{\mathrm{A}}^{-}$ formation. The fine structure seen in the 600-670 nm region is due to the vibrational sideband structure built upon the $\mathrm{Chl}_{\mathrm{D} 1}$ shift.

Dithionite treatment of a PSII preparation is a wellestablished method of $\mathrm{Q}_{A}$ and cyt reduction (Cox et al. 2009). In addition, the dithionite treatment causes the disassembly of the $\mathrm{CaMn}_{4} \mathrm{O}_{5}$ cluster of the OEC. The dithionite reduction of $\mathrm{Q}_{\mathrm{A}}$ in $C$. merolae PSII was confirmed by absorption changes observed in the $\mathrm{Q}_{\mathrm{y}}$ region, which mimic those induced by photo-illumination (Fig. 1). The reduced forms of cyt $b_{559}$ and cyt $c_{550}$ absorb in the $550 \mathrm{~nm}$ region where pheophytin $\mathrm{Q}_{\mathrm{x}}$ excitations of $\mathrm{Pheo}_{\mathrm{D} 1}$ and $\mathrm{PheO}_{\mathrm{D} 2}$ also occur. In the dithionite-treated sample, the $\mathrm{Pheo}_{\mathrm{D} 1} \mathrm{Q}_{\mathrm{x}}$ transition was in its electrochromically-shifted position, creating a rather congested spectrum in the 550 $\mathrm{nm}$ region. In the corresponding MCD spectrum, both reduced cyts give rise to MCD A-terms. An A-term MCD feature has the shape of the derivative of the absorption profile of the band inducing the MCD (Piepho and Schatz 1983). The pair of A-terms derived from reduced cyt absorptions is clearly seen in Fig. 1 in this region. These enable an assignment of cyt $b_{559}$ to $554.5 \mathrm{~nm}$ and cyt $c_{550}$ to $545.7 \mathrm{~nm}$, providing better precision than previous room temperature measurements (Krupnik et al. 2013). 


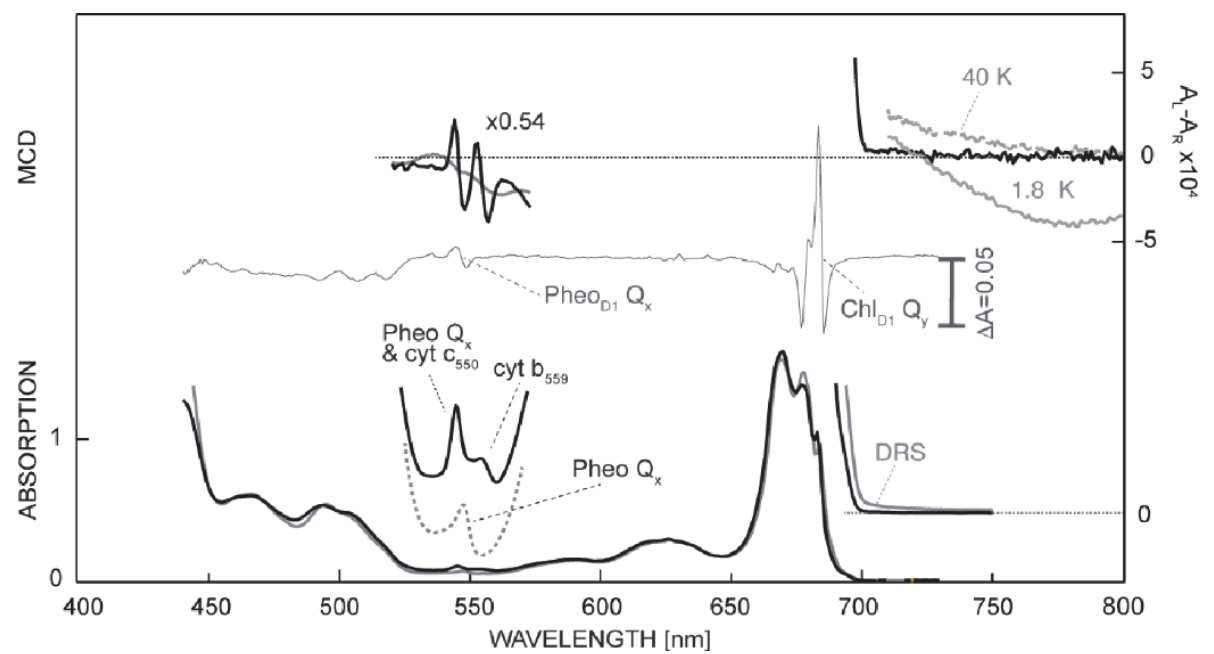

Fig. 1. Absorption (lower, left hand scale) and MCD (upper, right hand scale) of PSII cores derived from C. merolae. Black traces show dithionite-treated samples, whilst grey traces are samples not treated with dithionite. The absorption spectrum measured in the region between 440-730 nm was measured using a low OD sample, whilst the two detailed spectra between 520-590 nm and 690-750 nm were measured in a high OD sample (see text for definitions of the low and high OD). MCD were taken in an applied field of $5 \mathrm{~T}$. MCD of the cyt region around $550 \mathrm{~nm}$ was performed on a low OD sample. MCD of the DRS was measured with a high OD sample. The central thin grey trace shows the change in absorption (scale indicated) upon illumination with green light with fluence of $\sim 1 \mathrm{~mW} \mathrm{~cm}$ ${ }^{2}$ for a period of $5 \mathrm{~min}$ at $1.8 \mathrm{~K}$. All spectra shown are at $1.8 \mathrm{~K}$ except for the non-dithionite-treated DRS MCD for which spectra at both $1.8 \mathrm{~K}$ and $40 \mathrm{~K}$ were measured as indicated.

Absorption and MCD spectra of the nonreduced sample in the post $700 \mathrm{~nm}$ region revealed the broad, weak absorption characteristic of the DRS as seen in S. oleracea and T. vulcanus. The DRS in C. merolae has $\varepsilon \sim 4,000 \mathrm{M}^{-1}$ $\mathrm{cm}^{-1}$ at $702 \mathrm{~nm}$, while the MCD has $\Delta \varepsilon$ of $\sim 13 \mathrm{M}^{-1} \mathrm{~cm}^{-1}$ at $5 \mathrm{~T}$ which is similar to the values of other organisms previously studied (Krausz et al. 2005, Morton et al. 2015), although the tail seems to extend further to the red in C. merolae. Thus, the DRS has similar absorption and MCD properties in higher-plants, cyanobacteria, and algae. Its function in PSII, if any, is not yet known. Significantly, treatment of the sample with dithionite removed the characteristic absorption of the DRS in the $700-730 \mathrm{~nm}$ region, as shown in Fig. 1.

The MCD of the DRS in the untreated sample shown in Fig. 1 was taken at $1.8 \mathrm{~K}$ and $40 \mathrm{~K}$. Between $20 \mathrm{~K}$ and $40 \mathrm{~K}$, the MCD in the near infrared region is dominantly temperature independent, as expected for the DRS. By contrast, MCD spectra of this region measured at $1.8 \mathrm{~K}$ to $20 \mathrm{~K}$ in C. merolae exhibited a strong negatively signed and markedly temperature-dependent MCD. This temperature-dependent signal was absent in the dithionitetreated sample. The MCD of the dithionite-treated sample shown in Fig. 1 was taken at $1.8 \mathrm{~K}$. We attribute the temperature-dependent signal seen in the $700-850 \mathrm{~nm}$ region to most likely a cyt-based MCD, which is not present in S. oleracea or T. vulcanus. This temperaturedependent MCD is too intense to be associated with $\mathrm{d}-\mathrm{d}$ transitions in the $\mathrm{Mn}_{4} \mathrm{CaO}_{5}$ cluster (Morton et al. 2014). Spectroscopic measurements of $\mathrm{PsbV}$ isolated from C. merolae and of PSII in which the $\mathrm{Mn}_{4} \mathrm{CaO}_{5}$ cluster has been removed would help clarify this matter and are currently being pursued. The opportunity also exists to search for $\sim 780 \mathrm{~nm}$ fluorescence from the C. merolae DRS. A broad emission from the DRS has been identified in both S. oleracea and T. vulcanus (Morton et al. 2014).

The disappearance of the DRS state of C. merolae upon dithionite reduction may be attributed to its charge transfer characteristic. The excitation energy of an opticallyallowed charge transfer state is exquisitely sensitive to its dielectric environment. An indication of the extreme sensitivity of the DRS to changes in PSII is the drop in intensity of the DRS of $30 \%$ upon (photo-illumination induced) $\mathrm{Q}^{-}$formation (Hughes et al. 2007). It has also been noted (Krausz et al. 2008) that isolated RCs (Nanba and Satoh 1987) of PSII D1D2/cyt $b_{559}$ - which are stripped of proximal antennae CP43 and CP47, and have no $\mathrm{Q}_{\mathrm{A}}$ or redox-active $\mathrm{Tyr}_{\mathrm{Z}}$ - show no equivalent DRS in either their RC-6 form (which contains $6 \mathrm{Chl} a$ ) or RC-5 form (in which a ChlZ is removed). The Chl $a$ Qy absorptions of the isolated RCs are blue-shifted compared to those seen in intact PSII. Accordingly, any DRS equivalent to that seen in functional PSII should be easily visible. It is likely that the removal of PsbQ', and $\mathrm{Q}_{\mathrm{A}}$ reduction conspire to produce a significant shift of the DRS to a higher energy, and thus into a spectral region where it is not easily seen amongst the more intense $\mathrm{Q}_{\mathrm{y}}$ absorptions of $\mathrm{Chl} a$ in PSII core complexes. Another possibility is that dithionite reduction leads to sufficient disruption to the PSII protein so that the electronic overlap of the special pair $\mathrm{P}_{\mathrm{D} 1}-\mathrm{P}_{\mathrm{D} 2}$ is diminished to a point where the optical intensity of the charge transfer state involving these two Chl $a$ molecules becomes inhibited. 
We have shown that the DRS, i.e. the lowest energy state of the RC of PSII in the (red algae) C. merolae, has properties entirely analogous to those previously seen for the DRS in (plant) S. oleracea and (cyanobacterium) T. vulcanus. The DRS was confirmed to be a universal characteristic of functional (i.e. oxygen-evolving) PSII. Dithionite treatment of PSII in C. merolae led to a dramatic reduction in the intensity of DRS absorption in the $700-730 \mathrm{~nm}$ range, drawing comparisons to the lack of

\section{References}

Adachi H., Umena Y., Enami I. et al.: Towards structural elucidation of eukaryotic photosystem II: Purification, crystallization and preliminary X-ray diffraction analysis of photosystem II from a red alga. - BBA-Bioenergetics 1787: 121-128, 2009.

Ago H., Adachi H., Umena Y. et al.: Novel features of eukaryotic photosystem II revealed by its crystal structure analysis from a red alga. - J. Biol. Chem. 291: 5676-5687, 2016.

Butler W.L., Okayama S.: The photoreduction of C-550 in chloroplasts and its inhibition by lipase. - BBA-Bioenergetics 245: 237-239, 1971.

Cox N., Hughes J.L., Steffen R. et al.: Identification of the QY excitation of the primary electron acceptor of photosystem II: CD determination of its coupling environment. - J. Phys. Chem. B 113: 12364-12374, 2009.

Enami I., Okumura A., Nagao R. et al.: Structures and functions of the extrinsic proteins of photosystem II from different species. - Photosynth. Res. 98: 349-363, 2008.

Hughes J.L., Cox N., Rutherford A.W. et al.: D1 protein variants in Photosystem II from Thermosynechococcus elongatus studied by low temperature optical spectroscopy. - BBABioenergetics 1797: 11-19, 2010.

Hughes J.L., Rutherford A.W., Sugiura M. et al.: Quantum efficiency distributions of photo-induced side-pathway donor oxidation at cryogenic temperature in photosystem II. Photosynth. Res. 98: 199-206, 2008.

Hughes J.L., Smith P., Pace R. et al.: Charge separation in photosystem II core complexes induced by 690-730 nm excitation at 1.7 K. - BBA-Bioenergetics 1757: 841-851, 2006.

Hughes J.L., Smith P.J., Pace R.J. et al.: Low energy absorption and luminescence of higher plant photosystem II core samples. - J. Lumin. 122-123: 284-287, 2007.

Ifuku K.: Localization and functional characterization of the extrinsic subunits of photosystem II: an update. - Biosci. Biotech. Bioch. 79: 1223-1231, 2015.

Krausz E.: Selective and differential optical spectroscopies in photosynthesis. - Photosynth. Res. 116: 411-426, 2013.

Krausz E., Cox N., Arsköld S.P.: Spectral characteristics of PS II reaction centres: as isolated preparations and when integral to PS II core complexes. - Photosynth. Res. 98: 207-217, 2008.

Krausz E., Hughes J.L., Smith P. et al.: Oxygen-evolving photosystem II core complexes: A new paradigm based on the spectral identification of the charge-separating state, the primary acceptor and assignment of low-temperature fluorescence. - Photoch. Photobio. Sci. 4: 744-753, 2005.

Krupnik T., Kotabová E., van Bezouwen L.S. et al.: A reaction
DRS absorption in (nonfunctional) D1D2/cyt $b_{559}$ PSII preparations. The utility of precision low-temperature absorption, absorption difference, and MCD spectroscopies is highlighted as being able to make assignments in spectrally congested regions. The $\mathrm{Q}_{\mathrm{x}}$ absorptions of pheophytins become distinguishable from overlapping cyt features near $550 \mathrm{~nm}$ and the DRS from $\mathrm{Q}_{\mathrm{y}}, \mathrm{Mn}_{4} \mathrm{CaO}_{5}$ based and cyt absorptions in the 700-900 $\mathrm{nm}$ region.

center-dependent photoprotection mechanism in a highly robust photosystem II from an extremophilic red alga, Cyanidioschyzon merolae. - J. Biol. Chem. 288: 23529-23542, 2013.

Morton J., Akita F., Nakajima Y. et al.: Optical identification of the long-wavelength (700-1700 $\mathrm{nm}$ ) electronic excitations of the native reaction centre, $\mathrm{Mn}_{4} \mathrm{CaO}_{5}$ cluster and cytochromes of photosystem II in plants and cyanobacteria. - BBABioenergetics 1847: 153-161, 2015.

Morton J., Hall J., Smith P. et al.: Determination of the PS I content of PS II core preparations using selective emission: A new emission of PS II at $780 \mathrm{~nm}$. - BBA-Bioenergetics 1837: 167-177, 2014.

Morton J., Chrysina M., Craig V. et al.: Structured Near-Infrared Magnetic Circular Dichroism spectra of the $\mathrm{Mn}_{4} \mathrm{CaO}_{5}$ cluster of PS II in T. vulcanus are dominated by Mn(IV) d-d 'spin-flip' transitions. - BBA-Bioenergetics 1859: 88-98, 2018.

Nanba O., Satoh K.: Isolation of a Photosystem II reaction center consisting of D-1 and D-2 polypeptides and cytochrome $b-559$. - P. Natl. Acad. Sci. USA 84: 109-112, 1987.

Nilsson H., Krupnik T., Kargul J. et al.: Substrate water exchange in photosystem II core complexes of the extremophilic red alga Cyanidioschyzon merolae. - BBA-Bioenergetics 1837: 12571262, 2014

Pettai H., Oja V., Freiberg A. et al.: Photosynthetic activity of far-red light in green plants. - BBA-Bioenergetics 1708: 311 321, 2005.

Piepho S.B., Schatz P.N.: Group Theory in Spectroscopy with Applications to Magnetic Circular Dichroism. Wiley-Interscience, New York, Chichester, Brisbane, Toronto, Singapore 1983.

Reimers J.R., Biczysko M., Bruce D. et al.: Challenges facing an understanding of the nature of low-energy excited states in photosynthesis. - BBA-Bioenergetics 1857: 1627-1640, 2016. Schlodder E., Lendzian F., Meyer J. et al.: Long-wavelength limit of photochemical energy conversion in photosystem I. J. Am. Chem. Soc. 136: 3904-3918, 2014.

Shen J.R.: The structure of photosystem II and the mechanism of water oxidation in photosynthesis. - Annu. Rev. Plant Biol. 66: 23-48, 2015.

Suga M., Akita F., Hirata K. et al.: Native structure of photosystem II at 1.95 A resolution viewed by femtosecond X-ray pulses. - Nature 517: 99-103, 2015.

Umena Y., Kawakami K., Shen J.-R. et al.: Crystal structure of oxygen-evolving photosystem II at a resolution of $1.9 \AA$. Nature 473: 55-60, 2011. 